$\Phi=$

\title{
Nuclear Imaging in Dentistry -A novel modality to explore
}

\author{
Dathar Sahithi $^{1}{ }^{*}$, Sudhakara Reddy $^{2}$, JyothirmaiKoneru $^{3}$, M.Preethi ${ }^{1}$, Guvvala Satheesh ${ }^{4}$ \\ ${ }^{1}$ Post graduate student, Department of Oral medicine and Radiology, Vishnu Dental College \\ ${ }^{2}$ Professor and Head of Department of Oral medicine and Radiology, Vishnu Dental College \\ ${ }^{3}$ Reader, Department of Oral medicine and Radiology, Vishnu Dental College \\ ${ }^{4}$ Post graduate student, Department of Periodontics and Implantology, Government Dental College and Hospital, Hyderabad \\ *Corresponding author E-mail:sahithi.dathar9@gmail.com
}

\begin{abstract}
Nuclear imaging is an exceptional branch of medical science that has been evolved in the latest decades in early diagnosis of a disease. The main underlying principle is that radioisotopes are injected in the body and emits gamma rays which are detected by special image receptors. Various nuclear imaging modalities include Scintigraphy, SPECT (Single photon emission computed tomography) and PET (Positron emission tomography) which can assess any functional changes that occur within a diseased cell. This article has essentially dealt with the fundamental principles of various nuclear imaging modalities and their applications in oral and maxillofacial region.
\end{abstract}

Keywords: Fusion Imaging; Nuclear Medicine; Positron Emission Tomography (PET); Scintigraphy; Single Photon Emission Computed Tomography (SPECT).

\section{Introduction}

Nuclear medicine is a branch or speciality of medical science and imaging that uses radionuclides/ radioisotopes and depends on the process of radioactive decay in the diagnosis and treatment of disease. It is a broad term that covers many imaging techniques with the common basis, since ionizing radiation originating within the body is detected and then imaged so as to determine either physiology or anatomy of the subject (Boloor et al. 2013).

Film radiography, diagnostic ultrasonography, Computed Tomography $(\mathrm{CT})$ and Magnetic Resonance Imaging (MRI) are commonly used morphologic imaging techniques, which mainly require a macroscopic anatomic change for information to be recorded by an image receptor. However, in some human diseases abnormal biochemical processes either occur without any evident anatomic change or occur prior to it. Nuclear imaging provides an avenue to the radiologist to assess such physiologic/biochemical changes and also assists in differentiating between metabolically dying or deceased cells and those which are actively metabolising (Sharma et al.2008). Another added advantage is that nuclear imaging procedures most often identify abnormalities very early in the progress of a disease long before they became apparent with any other diagnostic procedures (Boloor et al. 2013).

Nuclear medicine is the medical speciality using small amounts of radioisotopes or radiopharmaceuticals as tracers to diagnose the disease. These tracers when injected into the body, gets attracted to special organs, bones or tissues throughout the body, and they further emit characteristic radiations, which will be captured by special electronic instruments like scintillation or gamma camera, which are widely used in nuclear imaging (Boloor et al. 2013).

Commonly used nuclear medical imaging modalities are Scintigraphy, SPECT (Single photon emission computed tomography) and PET (Positron emission tomography). Molecular imaging using high-resolution single-photon emission computed tomography (SPECT), and positron emission tomography (PET) has ad- vanced elegantly and steadily gained importance and eventually has held an assurance in the diagnostic, clinical and research arenas (Mushtaq et al.2012). These modalities can also reconstruct three dimensional (3D) images of the structures and thus have improvised the diagnostic efficacy, especially in the field of oncology.

The applications of nuclear medicine are poorly understood with respect to dentistry. This article thus emphasizes on the fundamental principles of various nuclear imaging modalities and their applications in oral and maxillofacial region.

\section{Various nuclear imaging techniques and their applications in oral and maxillofacial region}

\subsection{Scintigraphy}

The word "Scint" in Latin refers to "spark". It is a form of diagnostic test that is used in nuclear medicine, wherein radioisotopes are taken internally, and the emitted characteristic radiation is captured by external detectors called as gamma cameras so as to produce two-dimensional images.

In the latest era, the application of scintigraphy in bone and salivary glands scanning has been evolved and has accomplished a great importance. The areas of increased uptake of radiotracer appear as so called "hot spot" and those with diminished or no uptake will be evident as "cold spot"(Ferreira et al. 2002).

Bone scintigraphy is a functional imaging technique which uses technetium-99m- Methylene Diphosphonate (Tc-99m-MDP) to attain the bone metabolism activity and has affinity for calciumrichhydroxyapatite crystals of bone. This Tc-99m-MDP undergoes 'chemisorption' and gets bound to the bone matrix (Kalayci et al.2010). The underlying principle being that Tc-99m-MDP tends to accumulate in areas of active or high bone turnover, depending 
on the degree of osteoblastic activity and presence of vascularisation (Kalayci et al.2010). The image acquisition includes capturing the gamma rays emitted by the radiotracer within the human body by means of a gamma camera (Ferreira et al. 2002).

Owing to this principle of bone scintigraphy, it may be of value to evaluate the process of bone healing around the dental implants. It is also sensitive to bone fractures, infections and primary or secondary bone tumors, as all these pathologies tend to trigger osteoblast activity. Increased uptake occurs due to increased bone metabolism and decreased uptake occurs due to diminished osteogenic activity of bone and also due to disrupted vascularity (Ferreira et al. 2002).

Intraosseous carcinomas may have originated from lesions of the oral mucosa, either arises from epithelial residues, or may develop as a result of malignant transformation of a benign lesion (such as a cyst or a tumor), or as secondary metastatic deposits from primary tumors at distant sites (Ferreira et al. 2002). Bone scintigraphy can assist in preoperative evaluation of the malignant lesions, and these lesions most frequently depict increased radiotracer uptake and thus appear as hot spot.

Fibro osseous lesions like Fibrous dysplasia and Paget's disease showed a focal alteration in bone remodelling (Gupta et al.2011, Shankar et al. 2013). Bone scintigraphy can be helpful in evaluating the extent as well as activity of these lesions.

Inflammatory lesions of the jaws such as osteomyelitis, traumatic injuries, periapical and periodontal lesions, as well as diseases of the Temporomandibular joint (TMJ) such as osteoarthritis show a variation in bone metabolism and show a positive bone scan image. Although periapical changes are more apparent in conventional radiographs, yet bone scan can aid occasionally, as the uptake of the tracer occurs prior to the radiographic alterations (Ferreira et al. 2002). Scintigraphy assists to discover early changes in the TMJ skeleton that may end up in causing joint disc abnormalities.

Salivary gland scintigraphy uses $99 \mathrm{mTc}$ pertechnetate $\left({ }^{99 \mathrm{~m}} \mathrm{Tc}\right)$ which can substitute for Chloride in Sodium or Potassium Chloride $(\mathrm{Na}-\mathrm{K}-\mathrm{Cl})$ salivary transport pump and hence aids in measuring the fluid movement in the salivary acinar glands (Arrago et al. 1987, Klutmann et al 1999, Knoll et al. 2012, Santos et al. 2010). Santos et al had described the monitoring of salivary glands through scintigraphy in one patient with Sjogren's syndrome before and after the administration of $1 \%$ pilocarpine. The results illustrated that scintigraphy with $99 \mathrm{mTc}$ pertechnetate had assessed the effected location, progression of disease activity and also the severity of the glands involved (Santos et al. 2010).

\subsection{Positron emission tomography (PET)}

It is a functional imaging technique that uses positron emitting radiotracers to study biological or metabolic processes that occur within the human body. The emergence of PET as a choice for diagnosis, tumor and nodal staging, monitoring the prognosis of therapy and assessment of recurrence in cancer has led to an increasing demand for advanced imaging technology (Townsend 2004, Kubicek et al.2010, Macapinlac H. 2005). In the latest decade, PET has broadened its domain and has attained great significance in areas of head and neck oncology.

The basic underlying principle is to detect positron emitting isotopes indirectly via the two $511 \mathrm{keV}$ photons created when a positron annihilates with an electron released from the surroundings. In PET procedure, a radiotracer is introduced into the body that emits positrons as it decays. The emitted positron interacts with an electron through positron annihilation and produces an antiparallel emission of two $511 \mathrm{keV}$ photons (Zanzonico 2004, J A K Blokland et al. 2002) as shown in figure. The line on which this annihilation event occurs is referred to as line of response (LOR) (Zanzonico 2004).

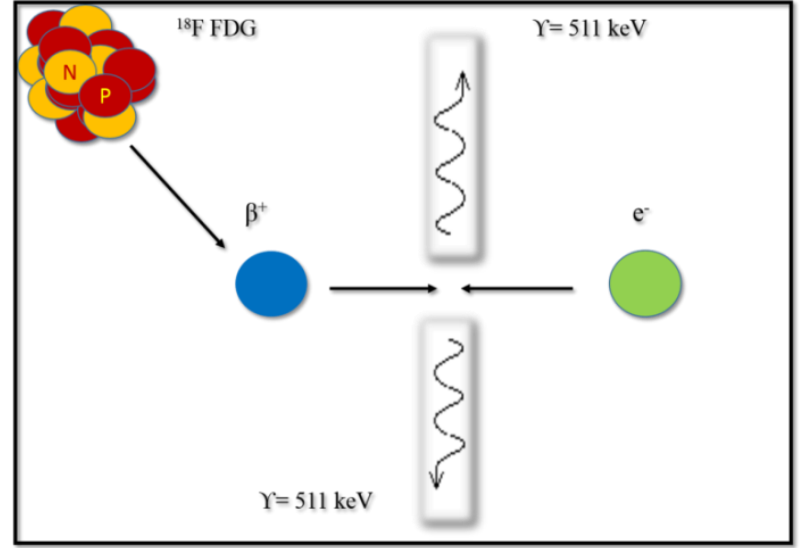

Fig. 1: Schematic representation of Annihilation reaction between Positron and Electron resulting in production of two $511 \mathrm{kev}$ photons anti parallel to each other.

At present, the most frequently used radiotracer in clinical PET scanning is fluorodeoxyglucose (FDG), which is an analogue of glucose that is labelled with fluorine-18(Townsend 2004). It is primarily used in PET because Fluorine-18 has a longer half-life (110 minutes) thereby permitting more flexibility in transport time to the effected sites and better administration to patients. However, FDG's widespread popularity is also due to its carrier component, glucose, a monosaccharide sugar (C6H12O6), which happens to be an essential circulating sugar in the blood. Malignant cells tend to have more glucose utilization due to up to regulation of hexokinase activity and have increased metabolic activity compared to adjacent normal cells (Kapoor et al.2004). FDG is taken up by metabolically active tumour cells and the rate of uptake of FDG by the tumour cells is directly proportional to their metabolic activity. Like glucose, it undergoes phosphorylation to form FDG6-phosphate, but does not proceed past the phosphorylation stage (hexose-6-phosphate) and further gets metabolically trapped within the cell which then gets detected during the PET procedure. Therefore, FDG-PET measures cellular glycolysis, which theoretically reflects proliferation and abnormal growth of the affected cells (Kapoor et al.2004).

In the modern era, FDG-PET has been illuminated in detection of the head and neck cancers at a very early stage and above all it can also identify the unknown and occult primary malignant tumours that undergo metastasis and manifest as secondary tumours elsewhere in the body. The detection rate with FDG-PET ranges from $10 \%$ to $60 \%$ and this difference mainly depends on patient selection and verification of strict clinical criteria (Macapinlac 2005).

FDG-PET has also explored its wings in evaluating increased blood flow activity in onlay grafts and regions of osteosynthesis, indicating bone repair in the graft (Boloor et al. 2013).

\subsection{Single photon emission computed tomography (SPECT)}

As the name suggests, SPECT uses single photon gammarayemission as the source of information, rather than X-ray transmissions as used in conventional Computed Tomography. SPECT is similar to PET in its use of radioactive tracer material and detection of gamma rays (Rahmim et al. 2008). In contrast with PET, the tracers used in SPECT emit gamma radiation, which is measured directly, whereas PET tracers emit positrons that annihilate with electrons up to a few millimeters away, causing two gamma photons to be emitted in opposite directions at $180^{\circ}$ to each other(Townsend 2004).

The application of SPECT in detection of head and neck cancer has also evolved and has gained significance in the recent decade. Nagamachi et al had investigated the usefulness of Thallium $201\left({ }^{201} \mathrm{Tl}\right)$ SPECT in 18 head and neck cancer patients and noted that all the primary tumours and cervical metastatic lymph nodes had shown increased ${ }^{201} \mathrm{Tl}$ uptake in both early and late images but 
did not show any correlation with the tumour size and its histological type(Nagamachi et al.1996).

Lee et al conducted a study to investigate the diagnostic efficacy of blood pool scintigraphy and SPECT in characterizing congenital vascular malformations (CVMs) in the head and neck area in a total of 154 suspected patients. The results described that SPECT is a useful method for classifying CVMs in the head and neck area due to its high diagnostic efficacy(Lee et al. 2010).

The temporo-mandibular joint(TMJ) is apparently evident using SPECT, when compared to double-dimension planar imaging, and can appreciate TMJ separately from the other parts of high bone density(Boloor et al. 2013).

\subsection{Fusion imaging (PET-CT, PET-MRI, SPECT-CT)}

Within its constraints, due to inherent characteristics of nuclear medicine images and their limited resolution power, it is being difficult to define the precise anatomical location of diseases (Marianiet al.2010). To overcome this limitation, the molecular and functional imaging provided by nucleotide imaging and anatomical imaging provided by CT/MRI have been merged into socalledHybrid/Fusion imaging.

SPECT provides some definite advantages over planar scintigraphy, and the additional localizing value of SPECT/CT is especially useful as a guide during surgical treatment. In particular, SPECT/CT is highly appreciated for its exact localization of ectopic glands by providing topographic correlation with adjacent normal anatomical structures(Mariani et al.2010).

Vermeeren et al had determined the additional value of single photon emission computed tomography with CT (SPECT/CT) for detection and localization of sentinel nodes in 38 patients with melanoma of the head and neck and has reported thatSPECT/CT had depicted an additional sentinel node in $16 \%$ of the patients and clearly showed the anatomic location of the nodes in all patients (Vermeeren et al. 2011).

PET/CT has also proved its utmost efficiency in diagnosis of head and neck carcinomas early in the course of the disease. However, it essentially aids in staging of the head and neck squamous cell carcinomas, detecting the unknown primaries and also monitoring the response to the treatment (Agarwal et al. 2008, Mathews et al.2011)

PET/MRI is a fusion imaging technology that incorporates both MRI soft-tissuemorphological imaging and PET functional imaging. Due to its limited availability, technical issues (especially regarding attenuation correction and the strong magnetic field that do not allow PET transmission scans to be implemented with positron-emitting rod sources), and the lack of clinical studies; PET/MRI cannot be regarded as an established modality in routine clinical practice, but still a promising technology, with its clinical and scientific value yet to be defined (Nensa et al. 2014). With the recent advancement of integrated whole-body PET/MRI and its application into the clinical settings, high accuracy in T-staging of tumour entities is being established. With regard to Nodal-staging, PET/MRI does not seem to be beneficial when compared with $\mathrm{PET} / \mathrm{CT}$ but provides similar $\mathrm{N}$-staging accuracy when applied as a whole-body staging approach (Buchbender et al. 2012).

Integration of functional and anatomic image data via union of PET/SPECT and CT/MRI provides additional clinically relevant information. It can be the inquest of choice for imaging because it can almost eliminate the false-positive and false-negative PET findings (Mathews et al.2011).

\section{Advantages and disadvantages of nuclear imaging}

The principal advantages of nuclear imaging is that functional images of the diseased tissues are obtained, whole skeletal system can be scanned within less span of time, recurrence can be easily detected and sensitivity is high, but it does have few limitations like the low specificity, it is rather expensive and is being contraindicated in pregnant and lactating women.

\section{Conclusion}

Nuclear medicine is an ideal specialty to adapt to the new discipline of molecular medicine, and it has extended its avenues into the field of dentistry. Nuclear medicine imaging techniques have been evolved as a routine diagnostic method to evaluate the osteoblastic activity around implants and in periodontal disease, also to identify the fractures, benign and metastatic tumors, bone grafts and TMJ disorders at a very early stage, and thus aids to bring about an intervention therapy. It also contributes in providing valuable information regarding localization of unknown primaries, cervical node metastases, staging of tumors, in detection of any recurrence and also provides independent prognostic information in patients undergoing cancer therapy.

Evolution of Hybrid imaging like SPECT/CT, PET/CT and PET/MRI has heralded a major progress in diagnostic radiology and has thus become a standard clinical imaging modality in patients with head and neck cancer. Fusion imaging is being substantiated to be more accurate rather than using individually in the detection and anatomic localization of head and neck cancers. Therefore, early diagnosis of the disease using this recent modality prevents its complications and unpredictable outcome in near future owing to the fact that "An ounce of prevention is better than a pound of cure".

\section{References}

[1] Agarwal V, Branstetter B, Johnson J. (2008) Indications for PET/CT in the Head and Neck. OtolaryngolClin N Am, 4: $23-$ 49.http://dx.doi.org/10.1016/j.otc.2007.10.005.

[2] Arrago JP, Rain JD, Brocheriou C, Rocher F. (1987) Scintigraphy of the salivary glands in Sjogen's Syndrome. ClinPathol, 40: $1463-$ 1467.http://dx.doi.org/10.1136/jcp.40.12.1463.

[3] Boloor V, Hosadurga R, Pratap S, Anupama R. (2013) Nuclear medicine in dentistry revisited: New avenues to explore; Clinical Cancer Investigation Journal, 2(3):189-194.http://dx.doi.org/10.4103/22780513.119253 .

[4] Buchbender C, Heusner T, Lauenstein T, Bockisch A, Antoch G. (2012) Oncologic PET/MRI, Part 1: Tumors of the Brain, Head and Neck. J Nucl 53:928938.http://dx.doi.org/10.2967/jnumed.112.105338.

[5] Ferreira RI, Almieda SM, Boscolo FN, Santos a, Camargo E (2002) Journal of Dental Education, 66(12):1381-1387.

[6] Gupta S, Umesh K, Warad NM, Ahmed S. (2011) Fibrous Dysplasia of Maxillary Bone: A Case Report. Al Ameen J Med Sci, 4 (1):92 -97.

[7] J a K Blokland et al. (2002) Positron emission tomography: a technical introduction for clinicians. European Journal of Radiology, 44: 70-75.http://dx.doi.org/10.1016/S0720-048X(02)00003-7.

[8] Kalayci A, Durmus E, Tastekin G, Isik K, Inan O (2010) Evaluation of osteoblastic activity around dental implants using bone scintigraphy. Clin. Oral Impl. Res, 21: 209-212.http://dx.doi.org/10.1111/j.16000501.2009.01822.x.

[9] Klutmann S et al. (1999) Quantitative Salivary Gland Scintigraphy. J Nucl Med Technol, 27:20-26.

[10] Knoll P, Krotla G, Bastati B, Koriska K, Mirzaei S.(2012) Improved quantification of salivary gland scintigraphy, Iran J Nucl Me, 20:5-10.

[11] Kapoor V, Mccook BM, Torok FS. (2004). an Introduction to PET-CT Imaging. Radiographics, 24: 523543.http://dx.doi.org/10.1148/rg.242025724.

[12]Kubicek G. (2010) FDG-PET staging and importance of lymph node SUV in head and neck cancer. Head \& Neck Oncology, 2(19):17.http://dx.doi.org/10.1186/1758-3284-2-19.

[13]Lee JY et al. (2010) Characterization of congenital lymphatic and Blood vascular malformations in the head and neck Sing blood pool scintigraphy and SPECT. Lymphology, 43: 149-157.

[14]Macapinlac H. (2005) FDG-PET in Head and Neck, and Thyroid Cancer; Chang Gung Med J ,28:284-95

[15] Mushtaq S. (2012) Role of nuclear medicine in dentistry. GJMEDPH, $1(6): 1-5$.

[16]Mariani G et al. (2010) A review on the clinical uses of SPECT/CT. Eur J Nucl Med Mol Imaging, 10.http://dx.doi.org/10.1007/s00259010-1390-8. 
[17]Mathews A, Jayakar JP, Rosenblat J. (2011) the role of FDG-PET and $\mathrm{PET} / \mathrm{CT}$ in the diagnosis and staging of head and neck cancer. UWOMJ, 80(2):29-31.

[18] Nagamachi et al. (1996). SPECT for evaluating head and neck cancer. Annals of nuclear medicine, 10(1):105-111. http://dx.doi.org/10.1007/BF03165062.

[19] Nensa F, Beiderwellen K, Heusch P, Wetter A.(2014) Clinical applications of PET/MRI: current status and future perspectives; DiagnIntervRadiol; 20:438-447.http://dx.doi.org/10.5152/dir.2014.14008.

[20] Rahmim A, Zaidi H. (2008) PET versus SPECT: strengths, limitations and challenges-Nuclear Medicine Communications, 29:193207.http://dx.doi.org/10.1097/MNM.0b013e3282f3a515.

[21] Sharma N, Neumann D, Macklis R. (2008) the impact of functional imaging on radiation medicine Radiation Oncology, 3 (25):113.http://dx.doi.org/10.1186/1748-717x-3-25.

[22] Shankar YU, Misra SR, Vineet DA, Baskaran P. (2013) Paget disease of bone: A classic case report. ContempClin Dent, 4: 227-30. http://dx.doi.org/10.4103/0976-237X.114858.

[23] Santos L et al. (2010) Scintigraphy of the salivary gland. Rev ClínPesqOdontol, 6(1):101-106.

[24]Townsend DW. (2004) Physical Principles and Technology of Clinical PET Imaging; Ann Acad Med Singapore, 33:133-45.

[25] Vermeeren L et al.( 2011) SPECT/CT for sentinel lymph node mapping in head and neck melanoma; Wiley Periodicals, Inc. Head Neck, 33:1-6.http://dx.doi.org/10.1002/hed.21392.

[26]Zanzonico P. (2004) Positron Emission Tomography: A Review of Basic Principles, Scanner Design and Performance, and Current Systems. Seminars in Nuclear Medicine, 34 (2): $87-$ 111.http://dx.doi.org/10.1053/j.semnuclmed.2003.12.002. 